\title{
Biofeedback and relaxation in the treatment of migraine headaches: comparative effectiveness and physiological correlates
}

\author{
J MICHAEL LACROIX, ${ }^{*}$ MELISSA A CLARKE, ${ }^{*}$ J CARSON BOCK, $\dagger$ NEVILLE DOXEY, $\dagger$ \\ ANNE WOOD, $\uparrow$ SANDRA LAVIS*
}

\author{
From Glendon College, York University* and The Ontario Workers' Compensation Board Hospital \& \\ Rehabilitation Centert, Toronto, Canada
}

SUMMARY Twenty-seven migraine headache patients were divided into three equal groups which received thermal biofeedback, frontalis EMG biofeedback, or relaxation training. Training was given under "massed" practice conditions (nine sessions per week) and consisted of 18 training sessions and six test-generalisation sessions. While improvements in headaches were observed in all groups, the best improvements took place in the thermal biofeedback group, which had almost complete elimination of migraine attacks by the end of training, and maintained that performance as long as six months after training. Examination of the patterns of skin temperature and EMG changes in the three groups over the course of training also points to a relationship between skin temperature control and reduction in migraine headache symptomatology, and suggests that this relationship is worthy of further investigation.

In the last decade thermal biofeedback has received increasing attention as a modality for treatment of migraine headaches. ${ }^{1-3}$ Typically, improvements of clinical proportions have been reported in $50-70 \%$ of patients trained to increase their peripheral temperature, which has usually been recorded from the fingers. However, much of the evidence for the effectiveness of this treatment modality has come from clinical reports and single-group studies, ${ }^{4-7}$ and the few recent reports which have employed control-group designs have not provided evidence for the superiority of thermal biofeedback over traditional forms of relaxation training or to frontalis EMG biofeedback in the treatment of migraine attacks. ${ }^{3-11}$ Furthermore, one recent paper even suggests that any beneficial effects of thermal biofeedback training on migraine headaches probably stem from placebo effects, as patients given control training to decrease their skin temperature benefited as much as did patients trained to increase their skin temperature.$^{12}$ However, the experimental evidence is not convincing. In most cases training

Address for reprint requests: Dr J Michael Lacroix, Department of Psychology, Glendon College, York University, 2275 Bayview Avenue, Toronto, Ontario, Canada, M4N 3M6.

Received 8 June 1982 and in revised form 26 December 1982. Accepted 17 January 1983 has been limited to a dozen sessions or less. ${ }^{810-13}$ Moreover, none of the previous experiments has provided evidence that subjects trained specifically to produce increases in skin temperature did in fact learn to produce such increases and to maintain them under conditions where feedback was withdrawn. Indeed, in at least two of the previous investigations subjects who received thermal training did not even show control of skin temperature when feedback was present. ${ }^{9}{ }^{10}$ It is at least possible that thermal biofeedback may not have been more effective than control treatments in these investigations simply because the subjects never learned sufficient control of the response to be able to generalise that control to the sorts of conditions (that is no feedback, real-life conditions) where migraine headaches are likely to occur.

In sum, the comparative effectiveness of thermal biofeedback and other therapeutic modalities in the treatment of migraine attacks remains a matter of contention, and constituted the first issue of concern in the present experiment. A three-group design was used, in which some migraineurs received biofeedback training to increase skin temperature, others received biofeedback training to decrease frontalis muscle EMG, and others still received a comparable amount of practice in progressive relaxation training techniques. Moreover, generalisation was pro- 
grammed through the use of no-feedback periods at the beginning and at the end of each training session, and through the use of no-feedback sessions interspersed within the training sessions.

The experiment departed from previous studies in two other important respects. First, the patient training was carried out under "massed" rather than "distributed" practice conditions. A review of the literature indicates that biofeedback training appears always to have been provided in weekly or bi-weekly sessions over a period of several weeks or months. This type of training is most typical of therapeutic interventions practiced by private practitioners or on an out-patient basis, and leaves open the question of whether more intense though less prolonged training, such as may be carried out on an in-patient basis, may or may not be equally effective. Second, most of the research participants in this study were compensation patients hospitalised on a short term basis for injuries suffered in a job-related accident. Whether for these patients migraine headaches can be sufficiently divorced from their other symptoms to be treated independently by biofeedback or relaxation is an issue that has not yet received much attention in the literature, where the strategy has usually been to find "pure" headache patients for treatment outcome studies.

In addition to these issues, the present experiment sought to generate information on the mechanisms through which thermal biofeedback, EMG biofeedback, and relaxation training may lead to improvements in the patient's migraine attacks, and on whether these mechanisms may. be different for improvements brought about through the three target treatments. To this end, recordings of both peripheral skin temperature and frontalis EMG were obtained from all patients throughout training and were related to changes in headache characteristics and to the treatment variables. The psychophysiological mechanisms through which biofeedback and other behaviourally oriented therapies effectuate changes in migraine headaches have as yet received little attention in the literature. However, those few studies which have systematically examined the relationship between target physiological responses in patients receiving biofeedback training and changes in headache characteristics have failed to observe systematic relationships. This has led to suggestions that thermal and EMG biofeedback may lead to changes in migraine symptomatology by training patients in general relaxation skills or by affecting their perceived mastery over their environment, and not by virtue of affecting specific migraine-related physiological response systems. $^{89}$ Insofar as improvements in headaches resulting from thermal or EMG biofeedback in the present study may be attributed simply to general relaxation or to placebo effects, ${ }^{12}$ it was expected that similar patterns of temperature-EMG-headache relationships would be observed in the three experimental groups.

\section{Method}

Subjects

Participants in the study consisted of 23 in-patients and four staff members at the Ontario Workers' Compensation Board Hospital and Rehabilitation Center. This facility is used exclusively in the assessment and treatment of individuals who have undergone industrial accidents in Ontario. The 23 in-patients therefore suffered from some accident-related medical problems (other than migraine attacks) for which they were receiving treatment at the time of their participation in the experiment. Participation in the research came through referral by a staff physician, with a diagnosis of migraine headache, and subsequent verification of that diagnosis by both a physician (we thank $\mathrm{Dr}$ $R$ Johnston, who served as our consultant in this capacity) and a psychologist expert in the diagnosis of headaches. Diagnosis was carried out in accordance with the criteria specified by Diamond and Delassio. ${ }^{20}$ Notwithstanding the research participants' compensation-related injuries, they appeared in other respects similar to the "pure" migraine headache patients who have been the focus of other studies. They comprised seven males and 20 females, with a mean age of 41.4 years (SD $=9.57$ years), and at the time of their introduction to the study they had suffered with migraine attacks for a mean of 19.5 years $(S D=13.2$ years). There were no significant group differences with respect to either of these variables. None of the subjects had had any experience with biofeedback or relaxation training for treatment of migraine headaches. Diagnosis was in accordance with the criteria specified by Diamond and Delassio. ${ }^{20}$

\section{Electrophysiological equipment}

Skin temperature was recorded from the distal phalange of the middle finger of the nondominant hand, through a Thermal Feedback System monitor Model DT-100 (BioFeedback Systems, Boulder, Co.). Where temperature feedback was provided, it consisted of a visual digital display of the patient's skin temperature, in degrees Fahrenheit. EMG was recorded from the frontalis using a standard 3-electrode assembly (recording surfaces were $18 \mathrm{~mm}$ in diameter), through a Med General EMG Feedback Module ( $\mathrm{N} \mathrm{102)}$. Where feedback was provided, it consisted of the visual meter display.

\section{Procedure}

Following the diagnostic interview with the psychologist, potential research participants were given a brief summary of the vascular theory of migraine (cf ref 14). In that context, they were also given the rationale that the training which they would undergo sought to teach them to inhibit the initial vasoconstrictive phase and thereby to prevent the development of the vasodilation that produces the headaches. The treatment was described as relatively new but as having been found successful in leading to significant 
improvements in 50-70\% of migraineurs. The multi-group comparison aspect of the study was not referred to, and no indications were given that other patients might be receiving different treatment modalities; potential research participants were simply told that the experiment sought scientific confirmation of what was already known clinically. If the patient agreed to participate, training began the following day. Assignment to groups was based on order of referral. The training regimen included 18 training and six test sessions. Patients came to the laboratory twice a day, once in the morning and once in the afternoon, from Monday to Thursday, and once on Friday. The two sessions on Day 1 were test sessions. Subjects then received a week's training ( 9 sessions), followed by two more test sessions, then another week's training, and two final test sessions. Test and training sessions were approximately $20-25 \mathrm{~min}$ in duration. On training sessions, patients were instructed to relax and, in the thermal and EMG biofeedback groups, to try to warm their hands or to relax their forehead muscles, respectively. For subjects in the two biofeedback groups, the relevant electrodes were then applied, and the acceptability of electrophysiological recordings was ascertained. The patient was then given one further minute to habituate, and a $1 \mathrm{~min}$ test sample of frontalis EMG or finger temperature was then obtained from subjects in the EMG and thermal biofeedback groups, respectively, during which feedback was not available. EMG was sampled seven times during that minute, at $10 \mathrm{~s}$ intervals, while finger temperature was sampled twice, at the beginning and the end of the 1 min test period. Subjects then practised their assigned task for $15 \mathrm{~min}$, with feedback available to subjects in the biofeedback groups, and this was followed by a 1 min test period, when EMG or finger temperature was again sampled as earlier. For subjects in the relaxation group, no physiological response was recorded on training days, and their training consisted of practice with a fixed series of relaxation tapes, which comprised breathing exercises and the Budzinzki series for limb heaviness and warmth and for facial relaxation. On test sessions, recordings of both finger temperature and frontalis EMG were obtained from all subjects, and no feedback was provided to subjects in either biofeedback group, nor were relaxation tapes presented to subjects in the relaxation group. Subjects in all groups were encouraged to practise their newly developed skills outside the laboratory/therapy situation, although no requirements were imposed with respect to such practice, and the subjects were not permitted to borrow either biofeedback equipment or relaxation tapes for that purpose.

Pre-treatment headache characteristics were determined initially on the basis of the patients' responses to structured interview questions. The same questions were asked again at the completion of treatment, and the patients were contacted by telephone for follow-ups 6-8 weeks, and again 6 months after treatment. Data from one or both follow-ups could not be obtained on three of the patients (two in the relaxation group and one in the temperature group). Because of the large variability between patients with respect to objective headache characteristics, and because of the possibility that different characteristics of the migraine attacks might be affected in different patients, a global 5-point self-rating scale of improvement was adopted as the primary indicant of therapeutic success (where 1 = no change, 2 = slightly improved, 3 = moderately improved, $4=$ greatly improved, and $5=$ symptom free).

The use of such a global scale appears warranted by the fact that, while the correlations between self-ratings and changes in headache frequency were significant at all posttraining measurement points $\left(r_{s}=0.39,0.51\right.$ and 0.47$)$, headache frequency changes accounted only for between 15 and $26 \%$ of the variance in self-rating scores.

\section{Results}

\section{Headaches}

Figure 1 presents the major results of the experiment with respect to the patients' headaches, in terms of the global assessment of the effectiveness of training provided by the rating scale. Figure 1 indicates that the group receiving thermal biofeedback improved most of all initially and maintained these improvements as long as 6 months post-training. Improvements of lesser proportions are also apparent in the other two groups at the end of training, with the relaxation group exhibiting further improvements in headaches over the six-month follow-up period, while the EMG biofeedback group remained stable over the same period. These observations were confirmed by an ANOVA employing groups and time as variates, which yielded a significant main effect of Groups $(F(2 / 24)=5 \cdot 25 ; p$ $<0.05)$ as well as a marginally significant interaction $(F(4 / 48)=2.41 ; p<0.07)$.

Figure 2 assesses the extent to which the global effects apparent in fig 1 may be attributed to changes in headache frequency: it displays the median headache frequency per month over the

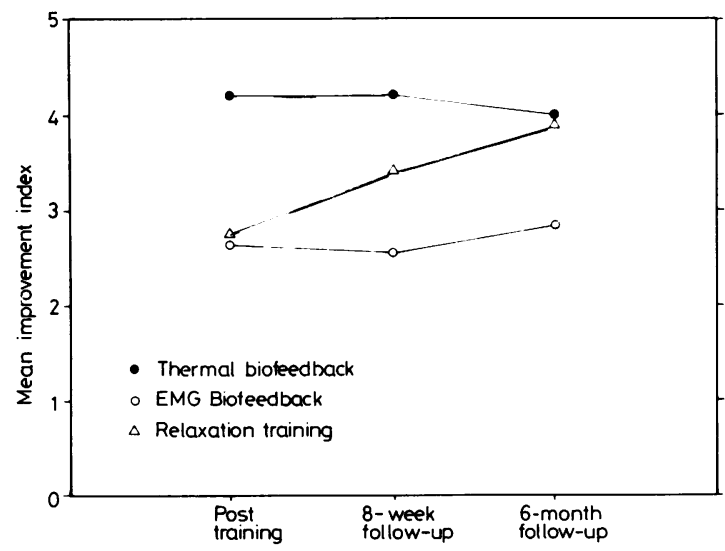

Fig 1 Mean improvement in patients' headaches in the three groups, as determined by the rating scale $(1=$ no change; 3 = moderately improved; 5 =symptom-free). 


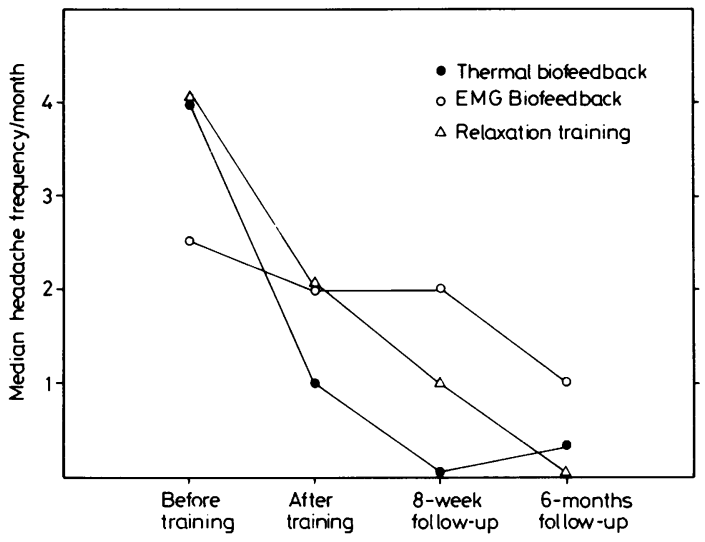

Fig 2 Median headache frequency per month in the three groups over the course of training and follow-up.

course of training and follow-up. The figure indicates, first, that headache frequency decreased in all groups from pre-training to post-training periods (main effect of time, $F(3 / 72)=7.28 ; p<0.01$ ). Second, fig 2 points to group differences in the rate of change in headache frequency, but also suggests that these might be obscured to some extent by initial differences between the groups. The posttraining, 8-week, and 6-month follow-up data were therefore analysed by an ANCOVA in which pretreatment headache frequency was used as the covariate. This analysis yielded a marginally significant interaction between Groups and Time $(F(4 / 47)=$ $2 \cdot 26 ; p<0 \cdot 10)$, which appears to reflect primarily the fact that headache frequency decreased considerably more in the thermal biofeedback and relaxation groups over the course of the follow-up period than in the EMG biofeedback group. Certainly, it is clear that by the 6-month follow-up, and even at the 8-week follow-up, most subjects in the thermal biofeedback and relaxation groups experienced few, if any, headaches. This substantial decrease in headache frequency makes it impossible to assess the extent to which decreases in headache intensity might also have contributed to the overall effects apparent in fig 1 . However, at the first post-training assessment, of five patients in the thermal biofeedback group still reporting headaches, all reported a decrease in intensity and also reported a decrease in medication intake. The comparable figures were three out of six and two out of six in the EMG biofeedback group, and two out of seven and none out of seven in the relaxation group. Of those patients who did not discontinue medication, there were no reports of any changes.

\section{Physiological changes}

Modification of physiological responses as a result of training might be manifested in terms of changes in the baseline levels of the response over days as a function of training, or in terms of an enhanced proficiency at effectuating phasic changes in the target response, or in both those ways. Figure 3 examines the former possibility, while fig 4 examines the latter. The left-hand panel of fig 3 depicts resting levels of skin temperature in the three groups, obtained during the initial test samples of the six test sessions. The right-hand panel of fig 3 depicts resting levels of frontalis EMG obtained similarly. While fig 3 suggests that resting skin temperature varied in the three groups over the course of training (Groups $\times$ Time interaction, $F(4 / 48)=2.92 ; p<$ $0.05)$, these changes do not appear systematic or sustained, and neither the main effect of groups nor that of time approached statistical significance (both $F \mathrm{~s}<1)$. Conversely, systematic decreases in resting EMG levels are apparent in all three groups over the course of training (Time main effect, $F(2 / 48)=$ $10.80 ; p<0.001$ ), and these are evident to about the same extent in the three groups (interaction $F<$ $1)$; group differences in resting EMG were not significant $(F(2 / 24)=1 \cdot 58 ; p>0 \cdot 10)$.

Figure 4 shows within-session changes produced in skin temperature over the course of training. The figure displays the performance of the three groups on the three test days, and also the performance of the thermal biofeedback group on training sessions from the first, middle, and last third of training. The figure suggests that the thermal biofeedback group was able to produce substantial increases in skin temperature on training days from the outset of training, and that biofeedback training resulted in generalisation of this performance to no-feedback test conditions. In keeping with this observation, a two-factor ANOVA of that group's performance, which employed Days and Test vs Training sessions as variates, yielded a significant interaction $(F(2 / 16)$ $=5.30 ; p<0.025)$. A comparison of the three groups' performance on test sessions yielded a significant main effect of days $(F(2 / 48)=4 \cdot 12 ; p<$ $0 \cdot 05$ ), reflecting the fact that increases in skin temperature were generally larger by the end of training than at the beginning, but the Groups $\times$ Days interaction did not approach significance. Homologous analyses of within-session changes in frontalis EMG failed to reveal any main effects or interactions which approached statistical significance: it appears that the changes which took place in EMG over the course of training were primarily in resting levels; within-session decreases in EMG were present but similar in the three groups and on test and training trials in the EMG biofeedback group, and 


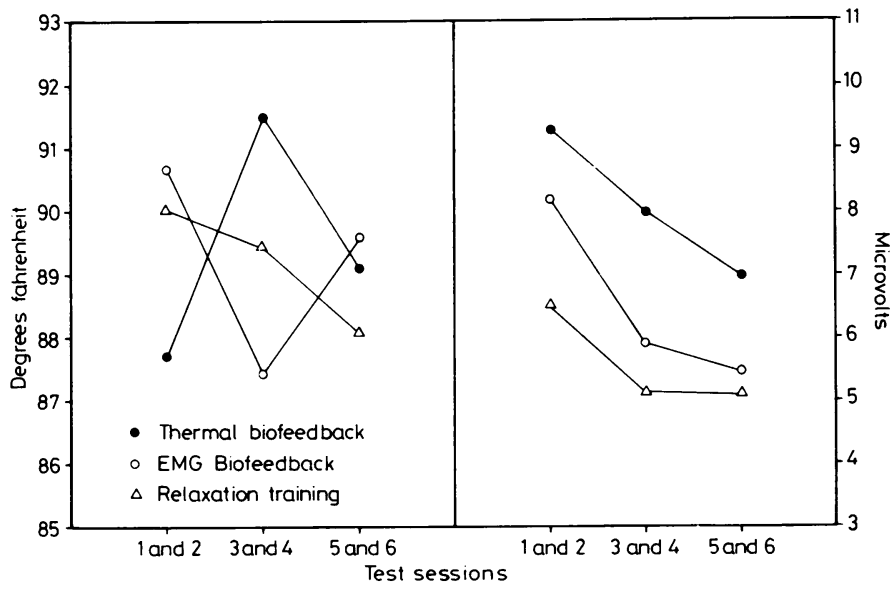

Fig 3 Mean resting levels of skin temperature (left-hand panel) and frontalis EMG (right-hand panel) in the three groups on pairs of test sessions given before training, half-way through, and at the end of training.

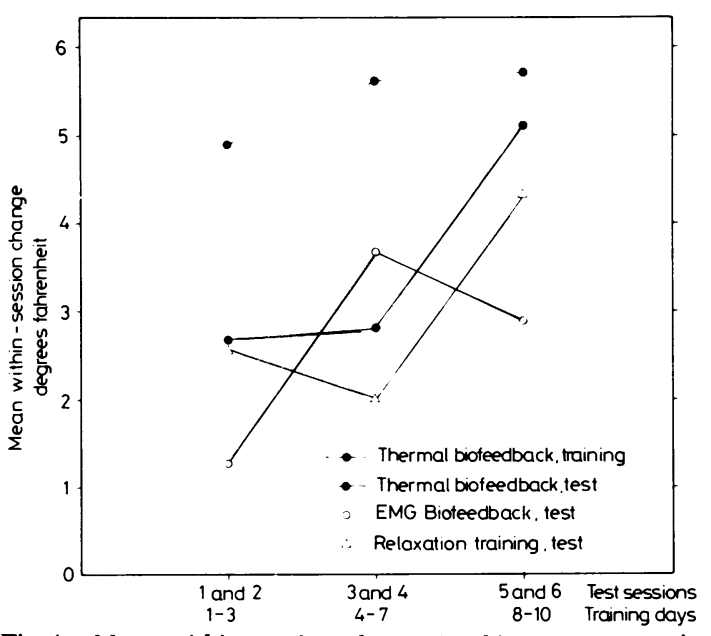

Fig 4 Mean within-session change in skin temperature in the three groups on pairs of test sessions given before training, half-way through, and at the end of training, and in the thermal biofeedback group on training sessions from the first, middle, and last third of training.

were not magnified as a function of training.

Finally, a number of product-moment correlations were computed in an attempt to determine the extent to which changes in the patients' headaches were related to changes in the two physiological responses of interest. Overall headache improvement, post-training and at 6-month follow up, as indicated by the rating scale, was correlated (a) with resting levels in skin temperature and frontalis EMG on the last two test sessions, (b) with the magnitude of within-session changes in the two physiological responses on the last two test sessions, (c) with changes in resting skin temperature and EMG level, between the first two and the last two test sessions, and (d) with changes in the magnitude of withinsession changes in the two physiological responses between the first two and the last two test sessions. All the correlation coefficients fell well short of significance.

\section{Discussion}

The experiment was addressed to two major issues, the comparative effectivenes of thermal biofeedback, EMG biofeedback, and relaxation training in the treatment of migraine headaches and the mechanisms through which these three treatment modalities may lead to improvements in migraine headaches. With respect to the first of these issues, the findings of the experiment appear reasonably clear-cut. Although improvements were obtained in all groups as determined by both self-ratings and the more objective frequency data, the magnitude of these improvements and their temporal characteristics differed among the groups. Thermal biofeedback emerged as the treatment of choice. It led to immediate and substantial improvements in the patients' headaches, and these were maintained as long as six months after the end of training. EMG biofeedback led to much smaller improvements in the patients' migraine attacks initially and these were maintained at about the same level over the follow-up period. Finally, relaxation training led to similar, small initial improvements in the patients' headaches, but also led to continued improvement following the end of training, such that, by the six month follow-up these patients had improved to a level comparable to that of patients receiving thermal biofeedback.

This pattern of results is congruent with earlier reports of equivalence in the effects of thermal 
biofeedback and relaxation training, but also differs from these reports in suggesting that the effects of thermal biofeedback are immediate while those of relaxation training take months to develop..$^{8-10}$ The present results also differ from previous studies in the magnitude of the effects obtained in the thermal biofeedback and relaxation groups. Unfortunately, comparisons between the present and previously published investigations are rendered difficult by the fact that the various investigators have employed widely different dependent measures. The only exception is headache frequency, which has been reported in most (albeit not all) studies. In this respect, the improvements observed in the EMG biofeedback group in the present investigation (an immediate decrease of $20 \%$ in median headache frequency and of $40 \%$ by the six month follow-up) appear roughly comparable to those obtained in other studies in groups receiving thermal biofeedback, EMG biofeedback, or relaxation training. ${ }^{9-13}$ Only the Blanchard et al. study ${ }^{8}$ reported a markedly higher percent reduction in headache frequency (ca $52 \%$ and $70 \%$ at three month follow-ups in groups receiving thermal biofeedback plus autogenic training and relaxation training, respectively). However, even these figures are inferior to those observed in the thermal biofeedback group in the present investigation (a 75\% immediate reduction, and a $92 \%$ reduction by the six month follow-up) and to the six month follow-up data in the relaxation training group $(100 \%$ reduction in median headache frequency). It appears, then, that the effects of the training regimen were generally more substantial in this than in earlier work, and that differences between groups should be attributed to more effective thermal biofeedback and relaxation training procedures rather than to less effective EMG biofeedback training, in this than in earlier studies.

It might be argued that the generally positive results obtained in this study are attributable to demand characteristics inherent in the use of selfreport measures, and that more objective headache measures would have led to much smaller improvements. However, such an assertion would be hardpressed to account for the present findings of differences between treatment groups. Moreover, compensation patients are usually considered more refractory to treatment than non compensation patients because of "compensation neurosis" ${ }^{15}$ - an assumption which our findings dispute, at least with respect to migraine attacks. Moreover, the kind of self-report measure employed here has been reported to correlate highly significantly with headache diary measures. ${ }^{16}$

The differences between the present findings and those previously reported in the literature, then, draw attention to the differences in procedures that may have led to larger and more immediate improvements in migraine headache characteristics by migraineurs receiving thermal biofeedback training in this experiment, and possibly also to more effective relaxation training. Three major differences are apparent. First, in most previous studies training has been limited to a dozen sessions or less, ${ }^{8} 0^{-13}$ as compared with twice that number in the present work. Second, in the present investigation, training was carried out under "massed" rather than "distributed" practice. Training for only a few sessions, separated by several days, may well be adequate in fostering the development of skills relevant to control of physiological functions in normal individuals. However, the acquisition of these skills may well be more difficult, and indeed may proceed differently, in individuals in whom the target physiological functions are in some way abnormal. ${ }^{1718}$ Finally, in the present investigation, generalisation was programmed through the use of no-feedback periods at the beginning and the end of each training session, and by the use of test sessions. Behaviour modifiers have learned that generalisation of learned behaviour should only be expected where such generalisation is specifically planned for. ${ }^{19}$ There is no reason to expect control of physiological functions to differ in this respect.

In addition to examining the comparative effectiveness of different treatment modalities for the treatment of migraine attacks, the present experiment sought to generate information on possible mechanisms through which the different treatment modalities exert their effects. The pattern of group differences with respect to headache changes argues that more is going on, at least in the thermal biofeedback group, than effects of a "general" nature. $^{891215}$ While general relaxation skills appear effective in the long run in controlling migraine headaches, as evidenced by the long-term effects observed in the relaxation training group, these skills seem to require some months to come to fruition. Placebo effects or effects attributable to enhancing the migraineurs' mastery over their environment might account for the improvement observed in the EMG biofeedback group (note that subjects in this group experienced "success" at the task in terms of decreases in EMG over days). However, these sorts of effects would not account for the additional improvements in the thermal biofeedback group, since these two groups differed only with respect to the response that was the target of biofeedback training. Moreover, it is unlikely that the better performance of the thermal biofeedback group might be attributable to that treatment modality being in some way more "credible". Recording 
EMG from the forehead and skin temperature from the fingers, intuitively, should grant EMG biofeedback treatment more face validity. Furthermore, we recently surveyed 46 undergraduate students and asked them which of the three treatments employed here they thought would prove most effective. The majority $(61 \%)$ believed that relaxation training would be the most effective, and the remainder favored the two types of biofeedback equally. This pattern held true, not only for the group as a whole, but also for those who claimed to suffer from migraine headaches or to be particularly knowledgeable about migraines.

Thus, the experimental data point to a relationship between skin temperature training and decreases in migraine attacks, over and beyond effects of a general nature. Furthermore, insofar as what was acquired during the course of training by subjects in the thermal biofeedback group was an ability to modify skin temperature on a phasic (within-sessions) rather than tonic (betweensessions) basis, it seems reasonable to suggest that what thermal biofeedback imparts immediately is an ability to abort headaches early in their development, rather than producing a shift in skin temperature resting levels (and, presumably, vasomotor activity) to the point where headaches are unlikely to occur at all. Whether a shift in resting levels does take place in migraineurs over a longer period, and whether this is what accounts for the long-term effects of relaxation training in the present study, are questions for further research.

One last point deserves comment. The argument for a specific relationship between skin temperature and migraine headaches is clearly at variance with the fact that the correlations obtained between the degree of improvement in patients' headaches as determined by the rating scale, and various measures of skin temperature control, were uniformly low. However, the correlations may have been limited to some extent by the restricted range of the rating scale. In addition, it is likely that the average magnitude of skin temperature changes is not as important for headache management as the consistency of these changes (unfortunately, there were not enough post-training test sessions in the present study for such correlations to be examined usefully). Finally, if it is possible to effectuate changes in headache characteristics independently through mechanisms involving phasic and tonic changes in skin temperature, as suggested above, correlations between phasic or tonic changes in skin temperature and headache changes are likely to be small unless account can be taken of the strategy (phasic or tonic) adopted by individual patients.

This research was supported by a grant from the
Ontario Workers' Compensation Board and by a grant from the Natural Sciences and Engineering Research Council of Canada (A0679) to JM Lacroix. Thanks are due also to the physicians at the WCB Hospital \& Rehabilitation Center, and particularly to Drs Wm. McCracken and R Johnson.

\section{References}

${ }^{1}$ Adams HE, Feuerstein M, Fowler JL. Migraine Headache: Review of Parameters, etiology, and intervention. Psychol. Bull 1980;87:217-237.

${ }^{2}$ Blanchard EB, Andrasik F, Ahles TA, Teders SJ, O'Keefe D. Migraine and tension headache: A metaanalytic review. Behaviour Therapy 1980;11:613-31.

${ }^{3}$ Turk DC, Meichenbaum DH, Berman WH. Application of biofeedback for the regulation of pain: A critical review. Psychol Bull 1979;86:1322-38.

${ }^{4}$ Mitch PS, McGrady A, Iannone A. Autogenic feedback training in migraine: A treatment report. Headache 1976;15:267-70.

s Sargent JD, Walters ED, Green EE. Psychosomatic self-regulation of migraine headaches. Sem Psychiatry 1973;5:415-28.

${ }^{6}$ Turin A, Johnson WG. Biofeedback therapy for migraine headaches. Arch Gen Psychiatry 1976;33: 517-9.

${ }^{7}$ Wickramasekera I. The application of verbal instructions and EMG feedback training to the management of tension headache-Preliminary observations. Headache 1973;13:74-6.

${ }^{8}$ Blanchard EB, Theobald DE, Williamson DA, Silver BV, Brown DA. Temperature biofeedback in the treatment of migraine headaches. Arch Gen Psychiatry 1978;35:581-8.

${ }^{9}$ Cohen MJ, McArthur DL, Rickles WH. Comparison of four biofeedback treatments for migraine headache: Physiological and headache variables. Psychosom. Med 1980;42:463-80.

${ }^{10}$ Lake A, Rainey J, Papsdorf JD. Biofeedback and rational-emotive therapy in the management of migraine headache. J Appl Behav Anal 1979;12: $127-40$.

$"$ Silver BF, Blanchard EB, Williamson DA, Theobald DE, Brown DA. Temperature biofeedback and relaxation training in the treatment of migraine headache: one-year follow-up. Biofeedback Self-Regul 1979;4: 359-66.

${ }^{12}$ Kewman D, Roberts AH. Skin temperature biofeedback and migraine headaches. Biofeedback Self-Regul 1980;5:327-45.

${ }^{13}$ Bild R, Adams HE. Modification of migraine headaches by cephalic blood volume pulse and EMG biofeedback. J Consult Clin Psychol 1980;48:51-7.

14 Delassio DJ. Wolffs Headache and Other Head Pain. New York: Oxford University Press, 1972.

${ }^{15}$ Kolb LC. Modern Clinical Psychiatry. Philadelphia: Saunders, 1977.

${ }^{16}$ Blanchard EB, Andrasik F, Neff DJ, Jurish SE, O'Keefe DM. Social validation of the headache diary. Behaviour Therapy 1981;12:711-5. 
${ }^{17}$ Lacroix JM. The acquisition of autonomic control through biofeedback: The case against an afferent process and a two-process alternative. Psychophysiology 1981;18:573-87.

${ }^{18}$ Qualls PJ, Sheehan PW. Electromyograph biofeedback as a relaxation technique: A critical appraisal and reassessment. Psychol Bull 1981;90:21-42.
${ }^{19}$ Baer DM, Wolf MW, Risley JR. Some current dimensions of applied behaviour analysis. J Appl Behav Anal 1968;1:91-97.

${ }^{20}$ Diamond S, Delassio DJ. The Practicing Physician's Approach to Headaches. 2nd ed. Baltimore: Williams \& Wilkins 1978. 